\title{
GEOMETRIC OBJECT BASED BUILDING RECONSTRUCTION FROM SATELLITE IMAGERY DERIVED POINT CLOUDS
}

\author{
Zhixin Li, Bo Xu, Jie Shan \\ Lyles School of Civil Engineering, Purdue University \\ 550 Stadium Mall Drive, West Lafayette, IN 47907, USA, \{li2887, jshan \}@purdue.edu, bobxu.purdue@gmail.com
}

KEY WORDS: Building reconstruction, Satellite images, Point cloud, Rectilinearization, Polygon decomposition, Primitive library

\begin{abstract}
:
3D building models are needed for urban planning and smart city. These models can be generated from stereo aerial images, satellite images or LiDAR point clouds. In this paper, we propose a geometric object-based building reconstruction method from satellite imagery derived point clouds. The goal is to achieve a geometrically correct, topologically consistent, and non-redundant 3D representation for buildings in urban areas. The paper first introduces our motivation, followed by a comprehensive review on related works. We then introduce the methodology and process developed in this paper. Primary results from the point clouds generated from WorldView high resolution satellite images are used to demonstrate the performance of the approach.
\end{abstract}

\section{INTRODUCTION}

$3 \mathrm{D}$ building model reconstruction is the process to create a geometrically correct, topologically consistent, and nonredundant $3 \mathrm{D}$ representation of a building. The inputs are often $3 \mathrm{D}$ point clouds derived from images or the ones collected by laser scanning techniques. Those point clouds provide abundant information for generating $3 \mathrm{D}$ building models. These building models can be a coarse representation of significant roofs, i.e., LOD2, or a detailed roof representation that includes fine objects, such as dormers, i.e., LOD3. Both can be in the standard format CityGML (Arefi et al., 2008). 3D building models have been widely applied in urban planning, environmental simulation, construction and mobile navigation. It has been studied in different disciplines, such as photogrammetry, remote sensing, computer vision, and surveying. This paper will be focused on using point clouds derived from satellite images to reconstruct LOD2 building models.

The library-based or model-based method fits buildings with predefined primitives (GEON, Geometric Icon). It estimates the model parameters and represent a single house model with a union of multiple GEONs. It is a powerful way to reconstruct complete and realistic building models. However, points cloud from satellite images often has high noise and irregular boundary. Incomplete or incorrect buildings often exist in the reconstructed results. To address this issue, we propose a rectilinear polygon decomposition method for GEON parameter estimation to achieve complete and regularized building models. Polygon decomposition or partition is to generate a set of primitive units from an input polygon. The resultant units do not overlap and their union equals the input polygon. Compared with using minimum bounding box, decomposing a polygon into GEONs and estimating their parameters will achieve a better quality and less over-estimation. To achieve this goal, we propose a workflow as follows. First, we generate building boundary directly from the segmented point cloud using the alpha-shape method. The irregular building boundary is then regularized to rectilinear boundary for decomposition. Finally, a shortest-edge-based rectilinear polygon decomposition (SHORTER) method, as we named, is proposed to get GEONs part by part. For each decomposed building part, a simple, predefined GEON will be estimated to achieve a best fit. After decomposition and estimation, a set of GEON parameters representing the building model are determined. To test the entire workflow and the proposed decomposition method, point clouds derived from stereo satellite images over University of California at San Diego (UCSD) area are used for generating building models. The point clouds are derived from a commercial stereo software system developed by Raytheon (Kalinski, 2014; Rathyeon) using co-collected panchromatic (PAN) and multispectral (MSI) WorldView3 (WV3) image pairs (Leotta et al., 2019). It is shown that we can achieve similar or even better reconstruction accuracy, compared with traditional reconstructed models.

\section{RELATED WORK}

Due to expensive acquisition cost of LiDAR data, many researchers developed methods to derive image point clouds with the help of aerial (Gehrke et al., 2010), UAVs (Rosnell and Honkavaara, 2012) and satellites (Shean et al., 2016) platforms. Since then, automatic generation of high-quality, dense point clouds from digital images by image matching has been proved to be a widely-used way for city reconstruction. However, researchers also found problems when processing image point clouds. For example, Shean and Alexandrow found that shadow edges appear due to the pixel-locking phenomenon (Shean et al., 2016). Furthermore, significant difference inherent to the LiDAR data - generally around trees and vegetation, where LiDAR in contrast to image point clouds has the ability to penetrate to the ground (Gehrke et al., 2010). Void areas in point clouds appear after processing and GEON-based method can use predefined model to fix the areas.

Many researchers have developed methods from diverse standpoints related to data, approaches, level of detail and so on. Improving geometrical details of a model is of great interest. They were more focus on geometry information and applied optimization method to get best fitting building models. Tutzayer and Haala used point clouds from mobile mapping system plus point clouds from dense image matching to enrich LOD2 building models and reconstruct facades (Tutzauer and Haala, 2015). They used colour information besides geometry to segment point cloud in problematic areas. Becker used LiDAR data and façade imagery for façade reconstruction (Becker and Haala, 2007). Window structures were extracted from LiDAR data and refined with overlapping images of façade. They both used RANSAC shape detection for detecting planar of roof and walls. However, planar roofs detected are irregular and need 
further process to generate closed models, such as through regularization and topologic calculation. However, GEONbased method will use less processes to find best model to fit point clouds. Regularized and compact models will be generated from respective regular primitives such as flat and gable roofs.

Compared with generating models from detected planar roofs, there are less researches on reconstructing building models based on primitives. Wang and Zhang proposed a decomposition method to decompose the compound buildings with symmetric roofs into semantic primitives by exploiting local symmetry contained in the building structure (Wang et al., 2015). Zhang proposed a primitive-based building reconstruction method with constraints using LiDAR and optical imagery (Zhang et al., 2011). However, in their approach, they need to recognize primitives in interactive mode and the primitive decomposition is not unique. Kada proposed a cell decomposition method to reconstruct buildings based on their primitives (Kada and McKineley, 2009). However, the number of cells can be large and redundant cells would exist in decomposition. Compared with those methods, our decomposition method will generate unique result with less primitives because of the boundary simplification process. Also, recognizing the type of a primitive is not needed because more basic GEONs are used to represent the buildings.

\section{METHODOLOGY}

This section will describe the workflow and methodology for fitting an irregular roof surface segmented from the input point clouds. We will take irregular roof polygon reconstructed from segmented image point clouds as input, then process it to fit GEONs in following steps. Firstly, from the input irregular polygon we calculate a rectilinear polygon. Then, we decompose it recursively based on shortest edge until all decomposed parts are rectangles. Finally, we use specific GEON (flat or shed) to fit each decomposed part. Figure 1 shows the work flow of the entire process

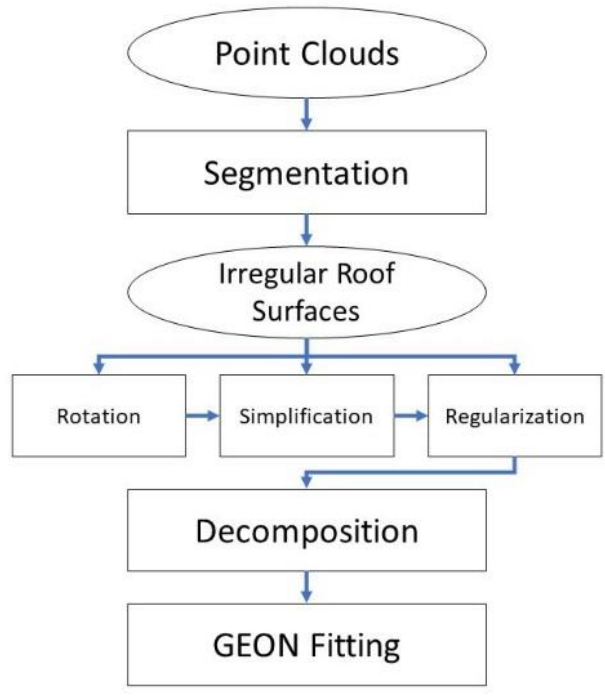

Fig 1. Workflow of shorted-edge-based polygon decomposition method (SHORTER) for building reconstruction

\subsection{Roof Surface Segmentation}

Extraction of building roof surfaces is necessary for reconstruction. In this process, we use the standard Random Sample Consensus (RANSAC) to derive the input surfaces from the point cloud generated through satellite images. This method iteratively and randomly selects samples with the least number of data points needed to determine the model parameters. It will stop when the number of data points from the rest of the data set fit with the parameters. The model parameters will be reestimated with the new set of data points. After data adjustment, building edges can be described accurately.

Those extracted surfaces provide initial information for the shape of the building (Bretar and Roux, 2005) and become the input to estimate the GEON parameters (Fu and Shan, 2017). However, because of shadow and occlusion, the point cloud from satellite images is not as satisfactory as LiDAR point cloud. For example, void areas in point cloud will make holes on the segmented surfaces. Limited resolution also causes irregular building edges which makes regularization necessary.

\subsection{Regularization}

Once segmented points for each roof surface are found, there will exist a crucial step to determine the boundary of the roof surface. In earlier researches, convex hull algorithm and its modified version has been a powerful way to determine the shape of a set of points. Convex hull algorithm works well on extracting roughly shape, but it may overestimate the shape of the points and result in a larger boundary. Recently, most studies use the alpha shape algorithm to trace a reasonable boundary as an initial approximate to the final shape. This method considers a parameter $\alpha$, responsible for selecting the straight segments that compose the object boundary (Xu and Harada, 2003). One critical problem is the determination of this parameter $\alpha$. A large $\alpha$ may result in a merged boundary where two planes are actually adjacent and a small $\alpha$ may split one complete boundary into several parts. As proposed by Jochem (Jochem et al., 2009) the average point spacing is a good estimate of the parameter $\alpha$ for building boundary extraction from LiDAR points. This method also has good performance for image-based point cloud has a uniform density. We use the alpha-shape algorithm for tracing roof polygons from segmented point clouds (Sampath and Shan, 2006). See Figure 2a for an example.

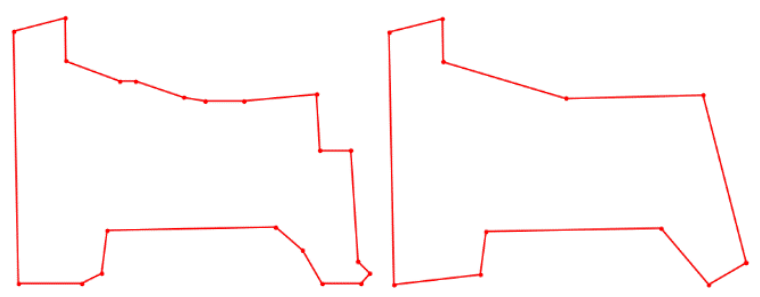

(a)

(b)

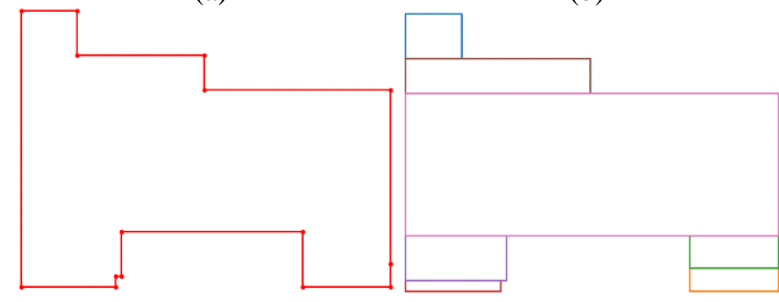

(c)

(d)

Fig.2 A building boundary represented by alpha shape (a), Douglas-Pecker (b), regularization (c), GEON decomposition (d). 
However, boundary extracted from alpha shape is not sufficient for GEON fitting because it is often irregular and has redundant vertices. Further refinement must be carried out before decomposition and fitting. Boundary simplification is used to remove its redundant vertices. One most popular method is the Douglas-Pecker algorithm (Ramer, 1971). Given a complex polyline, this algorithm will generate a similar curve with fewer points. Furthermore, with less vertices, polygon decomposition would likely avoid generating redundant parts. Figure $2 \mathrm{~b}$ shows an example from Douglas-Pecker simplification.

The first step for regularization is to find the dominant direction of the building boundary and rotate the coordinate system. To achieve this goal, we propose a projection density-based algorithm for estimating the dominant direction:

(1) Given a boundary of vertex (point) list $V=\left\{P_{1}, P_{2}, \ldots, P_{n}\right\}$, each point $P_{i}$ includes three coordinates: $\left(x_{i}, y_{i}, z_{i}\right)$

(2) Project all points onto $2 \mathrm{D}$ space;

(3) Generate candidate rotation angle set $\theta=$ $\left\{-90^{\circ}, \ldots,-1^{\circ}, 0^{\circ}, 1^{\circ}, \ldots, 90^{\circ}\right\}$

(4) For each angle in $\theta$, we rotate the coordinate system and calculate projection density. Then we find the angle $\theta_{d}$ that yields the smallest average projection density.

$$
\theta_{d}=\underset{\theta}{\operatorname{argmin}}\left(D_{\theta}\right)
$$

where $D_{\theta}=\max \left(\operatorname{mean}\left(D_{k x}\right), \operatorname{mean}\left(D_{k y}\right)\right)$

$$
\begin{aligned}
& D_{k x}=\sum_{i=1}^{n} \exp \left(-\frac{d_{x_{i}}^{2}}{\sigma^{2}}\right) \\
& D_{k y}=\sum_{i=1}^{n} \exp \left(-\frac{d_{y_{i}}^{2}}{\sigma^{2}}\right)
\end{aligned}
$$

$D_{k x}$ is the point density at $P_{i}$ along $x$ axis, $d_{x_{i}}$ is the distance from $P_{i}$ to $x$ axis.

Once the dominant direction $\theta_{d}$ is determined, we rotate the simplified polygon in Figure $2 \mathrm{~b}$ and apply a regularization step to create a rectilinear boundary. The criterion is to find the smallest rectilinear polygon that includes all initial vertices. For each oblique segmented line, we insert a vertex to generate a perpendicular line pair outside the input polygon. The method is developed as following steps:

(1) Given a clockwise 2D-boundary list $V=\left\{P_{1}, P_{2}, \ldots, P_{n}\right\}$, each point $P_{i}$ includes two coordinates: $\left(x_{i}, y_{i}\right)$;

(2) Generate line list $L=\left\{L_{1}, L_{2}, \ldots, L_{n}\right\}$;

(3) We extract oblique line segment in $L$ and for each line segment $L_{i}$ in $L_{\text {oblique }}$,

a. Calculate two insert point candidates based on two points $P_{i}$ and $P_{i+1}$ :

$$
\begin{aligned}
& P 1_{c}=\left[x_{i}, y_{i+1}\right] \\
& P 2_{c}=\left[x_{i+1}, y_{i}\right]
\end{aligned}
$$

b. For each candidate, we determine the relative position to $L_{i}$ based on equation as following:

$$
d=\left|\begin{array}{ccc}
x_{a} & x_{b} & x \\
y_{a} & y_{b} & y \\
1 & 1 & 1
\end{array}\right|
$$

where $x, y=$ candidate points

$x_{a}, y_{a}, x_{b}, y_{b}=$ vertex coordinates on line from $a$ to $b$. c. Since the order of polygon vertices is clockwise, a positive $d$ means candidate point is on the left of the line segment and a negative $d$ means point candidate is on the right side.

d. We test the relative position measurement $d$ for candidate points and keep point $P_{\text {insert }}$ with a negative $d$ which means this candidate is on the left of the line segment $L_{i}$.

e. Insert point $P_{\text {insert }}$ on line $L_{i}$ and update boundary list.

(4) Merge $L_{\text {oblique }}$ updated by inserting corner points and initial input boundary list $L$, then output new regularized boundary.

As an illustration to this procedure, Figure $2 b$ is regularized to form Figure 2c.

\subsection{Decomposition}

After the above step, we obtained a rectilinear polygon, which will be the input in this step. A partition of a polygon is a set of primitive units, which do not overlap and whose union equals the input polygon. Polygon decomposition is a problem of finding a partition which minimize certain objective function, for example a partition with a smallest number of units (Keil, 2000; Durocher and Mehrabi, 2012; Demir and Koperski, 2018; Li et al., 2019) or with units of smallest total side-length (Eppstein, 2010).

Polygon decomposition or polygon partition is an important subject in computational geometry. There are different kinds of decomposition problems, depending on the type of polygon being decomposed and on the types of primitives allowed. The most popular and well-studied polygon decomposition problem is to decompose a complex polygon to a set of triangles with smallest number. For a hole-free polygon with $n$ vertices, a triangulation can be calculated in time $O(n)$. And for a polygon with hole, the computational complexity is $O(n \log n)$ (Keil, 2000). Another problem is to decompose a rectilinear polygon (also called orthogonal polygon) into rectangles. Through all decomposition methods, rectilinear decomposition is the best way to restore the shape of an input building boundary since most buildings have a rectilinear boundary.

Building boundary should be represented with several sub primitives while the main primitives should be kept. Based on this criterion, we propose a shortest-edge-based algorithm (SHORTER) to decompose a rectilinear polygon into main parts and sub-parts. Firstly, we distinguish concave vertex and convex vertex on the rectilinear polygon (Lassak, 1993). For the shortest edge in the rectilinear polygon with one or more convex vertices, we derive a rectangle using the shortest edge and its neighbour edge. Then, we append the rectangle into a set consists of decomposed parts and cut it from the initial polygon. We go back to the second stage and recursively process the current shortest edge until the remaining part is a rectangle. Figure $2 \mathrm{c}$ is the polygon under decomposed and Figure 2d contain decomposed result. The algorithm is described as:

(1) Given a 2D clock-wise rectilinear polygon Poly and the vertex list $V=\left\{P_{1}, P_{2}, \ldots, P_{n}\right\}$, set an empty decomposition polygon set Poly $_{d}$

(2) For each vertex $P_{i}$ in $V$, determine the type of vertex and assign it to concave vertex set $V_{c c}$ or convex vertex set $V_{c v}$; 
(3) Generate line list $L=\left\{L_{1}, L_{2}, \ldots, L_{n}\right\}$

(4) For shortest edge $e_{A B}$ in $L$, get its vertices $P_{A}, P_{B}$, previous vertex $P_{A-1}$ and next vertex $P_{B+1}$

a. If $P_{A}$ in $V_{c c}$ and $P_{B}$ in $V_{c v}$ : append Poly $P_{\mathrm{A}, \mathrm{B}+1}$ in Poly $_{d}$

b. If $P_{A}$ in $V_{c v}$ and $P_{B}$ in $V_{c c}$ : append $P o l y_{\mathrm{A}-1, \mathrm{~B}}$ in Poly $_{d}$

c. If $P_{A}$ and $P_{B}$ are both in $V_{c c}$ : append Poly A, $\mathrm{B}+1_{1}$ in Poly $_{d}$ if $l_{\mathrm{A}-1, \mathrm{~A}} \geq l_{\mathrm{B}, \mathrm{B}+1}$, otherwise append Poly $_{\mathrm{A}-1, \mathrm{~B}}$ in Poly

where Poly $_{\mathrm{A}, \mathrm{B}}=$ rectangle polygon with diagonal $\overrightarrow{P_{A} P_{B}}$ $l_{\mathrm{A}, \mathrm{B}}=$ length from vertex $P_{A}$ to $P_{B}$

(5) Cut appended polygon from Poly and get rest polygon Poly $_{r}$, then return to step (2) and assign Poly as Poly .

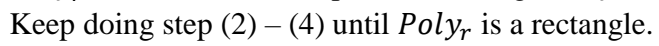

\subsection{GEON Fitting}

Given a set of decomposed parts, we use a cube GEON to fit the flat roof and trapezoid GEON to fit shed roof. More complicated roofs can be represented as a combination of several basic GEONs. For example, a gable roof can be represented as a combination of two trapezoid GEONs and a gambrel roof can be represented with a trapezoid GEON and a cube GEON. Common parameters for cube and trapezoid GEON include centroid, affine transformation and coordinate system parameters. Parameters for a cube GEON include length, width and height. For trapezoid, we add top surface normal as additional parameters. Those parameters are stored in JSON format and 3D building models can be rebuilt based on these parameters without any loss.

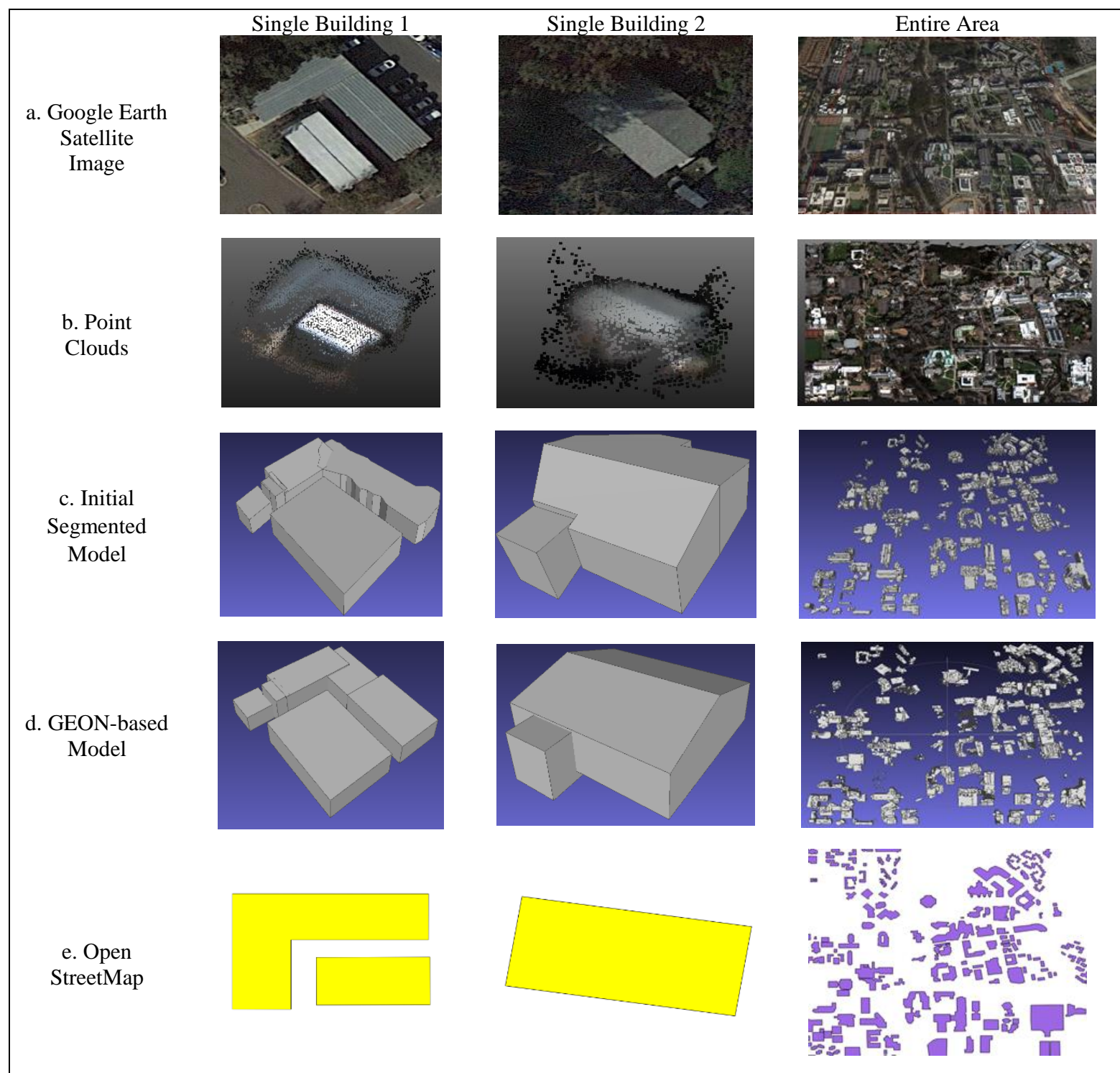

Fig 3. Sample building models from University of California at San Diego (from top to bottom: satellite image from Google Earth, point cloud, initial building surface, regularized and parameterized building models, OSM building boundary)

\section{EXPERIMENTS AND EVALUATION}

We use the point cloud over UCSD (University of California at San Diego) generated from satellite images to create both initial input building model and GEON-based model. We use building vector outlines from OpenStreetMap (OSM) to evaluate the quality. Since we don't have ground truth for structure of each 
building, we generated rasters of segmented models, GEONbased models and OSM building outlines. Take OSM as reference, we calculate the $2 \mathrm{D}$ correctness for segmented models and GEON-based models. Then we clip the point clouds within OSM building boundary and generated DSM to calculate 3D correctness. Evaluation result is listed in Table 1. See Figure 3 below for a few sample results from two complex buildings and the entire experiment area. Figure 3 a show the satellite images from Google Earth, and Figure 3b show the corresponding point clouds derived from the satellite images. Figure $3 \mathrm{c}$ presents the segmented roof surfaces models, and Figure $3 \mathrm{~d}$ are the reconstructed GEON models. Figure 3e shows the OSM building outlines. We can see after using perpendicular feature of building boundary, some missing parts are fixed through the process. Evaluation result is shown in Table 1.

\begin{tabular}{|c|c|c|c|}
\hline & $\#$ vertices & $\begin{array}{c}\% 2 \mathrm{D} \\
\text { Correctness }\end{array}$ & $\begin{array}{c}\% 3 \mathrm{D} \\
\text { Correctness }\end{array}$ \\
\hline Initial Model & 45206 & 83.6 & 82.1 \\
\hline GEON Model & 9764 & 84.6 & 83.8 \\
\hline
\end{tabular}

Table 1.2D and 3D correctness of initial segmented and GEONbased model in the UCSD area.

\section{CONCLUSION}

GEON-based building model reconstruction from satellite image-derived point cloud is addressed in a three-step process: segmentation, regularization (including boundary tracking and simplification) and decomposition. Segmentation gives initial input polygon and is a key step in the process. Using prior knowledge on buildings, such as perpendicular edges, our algorithm can fix missing part caused by shadow or occlusion in the point cloud. By decomposing segments polygon, the algorithm gives a best estimation result to fit the initial polygon. The decomposed parts are useful for generating building models with multiple levels of details. By reconstructing building models with decomposition and primitives, more realistic and complete models can be generated. Using point clouds derived from satellite images can save cost and time, compared with LiDAR data, and can cover a large area. Shorted-edge-based polygon decomposition method solves non-uniqueness and reduce the amount of decomposed parts, compare with cell decomposition. However, since we project all building vertices on the same direction, building with oblique parts may not be represented correctly. And some over-regularization boundaries also appear in reconstructed models. Future work will concentrate on import more types of primitives (including sphere, cylinder, shed roof, gable, etc) and non-rectilinear decomposition.

\section{ACKNOWLEDGEMENT}

This work is in part supported by the CORE3D Program of the Intelligence Advanced Research Projects Activity (IARPA). The views and conclusions contained herein are those of the authors. We would like to thank Dr. Matthew Leotta and Dr. Chengjiang Long of Kitware Inc. for their generous assistance in project management and related data processing.

\section{REFERENCES}

Andrzej, L., Christos, L., Jörg, S., 1987. "Algorithms for minimum length partitions of polygons". BIT Numerical Mathematics, Vol. 27, pp. 474-479.

Arefi, H., Engels, J., Hahn, M., Mayer, H., 2008. "Levels of Detail in 3D Building Reconstruction from LIDAR. ISPRS Archives of the Photogrammetry". Remote Sensing and Spatial Information Science, pp. 485-490.

Becker, S., Haala, N., 2007. "Refinement of building façades by integrated processing". IAPRS., PIA07, pp. 7-1.

Bretar, F., Roux, M., 2005. "Extraction of 3D planar primitives from raw airborne laser data: a normal driven RANSAC approach". Proceedings of IAPR Conference on Machine Vision Applications, Tsukuba, Japan, pp. 452-455.

Demir, I., Koperski, K., 2018. "DeepGlobe 2018: A Challenge to Parse the Earth through Satellite Images". IEEE/CVF Conference on Computer Vision and Pattern Recognition Workshops, pp. 172-17209.

Durocher, S., Mehrabi, S., 2012. "Computing Partitions of Rectilinear Polygons with Minimum Stabbing Number". Computing and Combinatorics. COCOON, Vol. 7434, pp. 228239.

Eppstein, D., 2010. "Graph-Theoretic Solutions to Computational Geometry Problems". Graph-Theoretic Concepts in Computer Science. Lecture Notes in Computer Science, pp. 116.

Fu, CS., Shan, J., 2017. "3D Reconstruction from Point Primitives: A Geometric Graph Approach". The International Archives of the Photogrammetry, Remote Sensing and Spatial Information Sciences, pp. 203-208.

Gehrke, S., Morin, K., Downey, M., Boehrer, N., Fuchs, T., 2010. "Semi-Global Matching: An Alternative to LiDAR for DSM Generation?". International Archives of Photogrammetry \& Remote Sensing, Vol. 38, pp. 2.

Jochem, A., Hofle, B., Rutzinger, M., Pfeifer, N., 2009. "Automatic Roof Plane Detection and Analysis in Airborne LiDAR Point Clouds for Solar Potential Assessment". Sensors., pp. $485-490$.

Kada, M., McKineley, L., 2009. "3D Building Reconstruction from LiDAR Based on A Cell Decomposition Approach". The International Archives of the Photogrammetry, Remote Sensing and Spatial Information Sciences 38 (Part 3/W4), pp. 47-52.

Kalinski, A., August 2014, "3d models from 2d imagery: Raytheon's intersect dimension". Geospatial Solutions.

Keil, J.M., 2000. "Polygon Decomposition". Handbook of Computational Geometry. pp. 491.

Lassak, M., 1993. "Approximation of convex bodies by rectangles". Geometriae Dedicata, Vol. 47, Issue 1, pp. 111-117.

Li, X., Lin, Y., Miller, J., Cheon, A., Dixon, W., 2019. "Primitive-based 3D Building Modeling", arXiv:1901.05554, https://arxiv.org/abs/1901.05554. 
Leotta, M.J., Long, CJ, Jacquet, B., Zins, M., Lipsa, D., Shan, J., Xu, B., Li, ZX, Zhang, Xu, Chang, S-F., Purri, M., Xue, J., Dana, K., 2019. "Urban Semantic 3D Reconstruction from Multiview Satellite Imagery", CVPR Workshop EARTHVISION: Large Scale Computer Vision for Remote Sensing Imagery, Long Beach, CA, USA, June 17.

Ramer, U., 1972. "An iterative procedure for the polygonal approximation of plane curves", Computer Graphics and Image Processing, Vol. 1, pp. 244-256.

Raytheon. Raytheon analytics: Intersect dimension. https://www.raytheon.com/capabilities/products/analytics.

Online; accessed 7 April 2019.

Rosnell, T., Honkavaara, E., 2012. "Point Cloud Generation from Aerial Image Data Acquired by a Quadrocopter Type Micro Unmanned Aerial Vehicle and a Digital Still Camera". Sensors, Vol. 12, Issue 1, pp. 453-480.

Sampath. A., Shan, J., 2006. "Building Boundary Tracing and Regularization from Airborne Lidar Point Clouds". Photogrammetric Engineering and Remote Sensing, vol. 73, Issue 7, pp. 805-812.

Shean, D.E., Alexandrov, O., Moratto, Z.M., Smith, B.E., Joughin, L.R., Porter, C., Morin, P., 2016. "An automated, Opensource Pipeline for Mass Production of Digital Elevation Models from Very-high-resolution Commercial Stereo Satellite Imagery". ISPRS. Journal of Photogrammetry and Remote Sensing, Vol. 116, pp. 101-117.

Tutzauer, P., Haala, N., 2015. "FAÇADE Reconstruction Using Geometric and Radiometric Point Cloud Information".

IAPRS, Munich, Vol. XL-3/W2, pp. 247-252.

Wang, H., Zhang, W., Chen Y., Chen M., Yan K., 2015. "Semantic Decomposition and Reconstruction of Compound Buildings with Symmetric Roofs from LiDAR Data and Aerial Imagery". Remote Sensing. Vol. 7, Issue 10, pp. 13945-13974.

Xu, X., Harada, K., 2003. "Automatic Surface Reconstruction with Alpha-shape Method". The Visual Computer. Vol. 19, Issue 7-8, pp. 431-443.

Zhang, W., Grussenmeyer, P., Yan, G., Mohamed, M., 2011, "Primitive-Based Building Reconstruction by Integration of LiDAR Data and Optical Imagery". International archives of photogrammetry and remote sensing and spatial information sciences. Vol. 38, pp. 7-12. 